\title{
Implementação do direito de participação das crianças em contexto de jardim de infância: As perceções dos educadores
}

\author{
Luísa Lopesi, Nadine Correiaii \& Cecília Aguiarii \\ ISCTE - Instituto Universitário de Lisboa, Portugal
}

\section{Resumo}

Com este estudo pretendeu-se investigar a relação entre a qualidade observada em contexto de jardim de infância e a promoção da participação, bem como avaliar as características psicométricas de uma medida de avaliação das perceções dos educadores de infância acerca da implementação do direito de participação das crianças em contexto de jardim de infância. Participaram 168 educadores de infância, sendo que, destes, 40 foram observados, em contexto de sala, com o Classroom Observation Scoring System (Pianta, La Paro, \& Hamre, 2008). Identificámos dois componentes das perceções dos educadores acerca da participação das crianças: Expressão e Responsabilidade das Crianças e Tomada de Decisão pelo Adulto. A Tomada de Decisão pelo Adulto estava negativamente associada à qualidade das salas e às habilitações académicas dos educadores. Paralelamente, educadores do setor público obtiveram resultados mais elevados no componente Expressão e Responsabilidade das Crianças do que educadores do setor privado com fins lucrativos. Os dados obtidos fornecem evidências que suportam, moderadamente, a fidelidade e a validade da medida.

Palavras-chave

Direito de participação das crianças; Questionário; Perceções; Jardim de infância 


\section{Introdução1}

A participação das crianças constitui um direito que se traduz na oportunidade de cada criança ser ouvida e de ter as suas opiniões tidas em consideração (Horwath, Hodgkiss, Kalyva, \& Spyrou, 2011). Expresso nos artigos $12 .^{\circ}$ e $13 .^{\circ}$ da Convenção dos Direitos da Criança (CDC) (UNICEF, 1989), este direito atribui aos adultos responsabilidades pela criação de condições para a sua implementação (Lansdown, 2001). No entanto, o pleno exercício do direito de participação das crianças não é, ainda, uma realidade nos vários domínios da sociedade (Horwath et al., 2011).

Os contextos de educação de infância constituem cenários de desenvolvimento onde as crianças devem poder efetivar o direito de participação e influenciar tudo o que Ihes diga respeito (Sheridan \& Samuelsson, 2001). Contudo, existem, ainda, poucas evidências empíricas sobre a forma como a participação das crianças é percecionada e implementada nestes contextos. O objetivo deste estudo é, precisamente, contribuir para colmatar tal lacuna, avaliando as características psicométricas de uma medida especificamente desenhada pelas autoras para avaliar a perceção dos educadores de infância acerca do grau de implementação do direito de participação das crianças em contexto de jardim de infância.

$\mathrm{Na}$ breve resenha de literatura que se segue propomo-nos identificar: (a) perspetivas teóricas sobre a participação, salientando a multidimensionalidade deste constructo; (b) benefícios da participação para o desenvolvimento da criança; e (c) paradigmas de aprendizagem e modelos pedagógicos que preconizam uma pedagogia da participação. Discutiremos, ainda, a participação enquanto indicador de qualidade de contextos de educação de infância.

\section{O direito de participação e a educação da infância}

Ao longo dos últimos anos, tem-se assistido, no campo sociológico e sobretudo da sociologia da infância, a uma reconceptualização da infância, defendendo-se a necessidade de considerar as crianças como atores sociais plenos, com direitos, dotados de competências, voz e ação próprias, integrados nos processos em que participam, e não apenas dependentes dos adultos. Esta nova forma de entendimento contribuiu para o reconhecimento 
da capacidade das crianças para influenciarem ativamente a sua vida, permitindo proclamar uma cidadania da infância e influenciando o surgimento de novas formas de desenvolver investigação (Prout \& James, 1997; Sarmento, Fernandes, \& Tomás, 2006; Soares, 2006).

Após a adoção da CDC (1989), Hart (1992) foi dos primeiros a teorizar sobre a participação enquanto direito, influenciando, de forma decisiva, não só o surgimento de abordagens e perspetivas subsequentes, como também o estabelecimento de programas e políticas internacionais. Tendo definido participação como o processo de partilha de decisões que afetam a vida das crianças e da comunidade onde estão inseridas, o autor estabeleceu oito níveis de participação - Manipulação, Decoração, Tokenismo (simbolismo), Atribuída mas informada, Criança consultada e informada, Decisões iniciadas pelo adulto e partilhadas com as crianças, Decisões iniciadas e dirigidas pelas crianças e Decisões iniciadas pelas crianças e partilhadas com os adultos -, sendo que nos três primeiros não existe participação efetiva.

A proposta de Hart gerou críticas, nomeadamente por descrever, essencialmente, o papel dos adultos (Reddy \& Ratna, 2002) e por se tratar de um modelo hierárquico, em que cada nível é qualitativamente superior ao anterior (Horwath et al., 2011). Consequentemente, outros investigadores (e.g., Kirby, Lanyon, Cronin, \& Sinclair, 2003; Shier, 2001; Treseder, 1997, cit. por Horwath et al., 2011) desenvolveram modelos alternativos. Por exemplo, Kirby e colaboradores (2003) propõem um modelo composto por quatro níveis: (1) As crianças e pontos de vista dos jovens são tidos em consideração; (2) As crianças e jovens são envolvidos na tomada de decisões; (3) As crianças partilham o poder e a responsabilidade pela tomada de decisão; e (4) As crianças tomam decisões autónomas. Neste modelo, nenhum nível é melhor do que outro. O contexto, as atividades, as decisões e os participantes é que determinam o nível adequado de participação. No domínio da sociologia da infância, Tomás (2007) sugere que o direito de participação pode ainda ser conceptualizado atendendo às suas diferentes dimensões: arenas de participação (i.e., os contextos públicos e privados enquanto espaços em que pode ser exercido, de diferentes formas), âmbitos de participação (i.e., a forma plena, circunstancial ou contínua, de carácter mais organizado ou mais espontâneo, permanente ou efémero, com que pode ser exercido), sentidos de participação (e.g., a medida em que se incita à 
promoção de atividades de defesa e divulgação dos direitos das crianças) e condições de participação (i.e., reconhecimento do direito de participar, capacidades para exercê-lo e meios para o efetivar).

Sendo o âmbito do conceito de participação tão vasto e transversal, não surpreende que o seu exercício possa ter impacto nos diversos sistemas da sociedade. Efetivamente, o exercício deste direito pode trazer benefícios para as crianças/jovens, para as organizações, para os decisores políticos e para a sociedade (Horwath et al., 2011). De um modo geral, os autores que se debruçam sobre este domínio têm identificado a autoestima (Gesellschaft für Technische Zusammenarbeit [GTZ], 2010; Kirby \& Bryson, 2002; Sandberg \& Eriksson, 2010) e a motivação (Kirby \& Bryson, 2002) como potenciais benefícios da participação. Adicionalmente, a investigação sugere que as crianças que se sentem envolvidas e valorizadas, através da participação, têm menos tendência para se envolverem em comportamentos de risco (GTZ, 2010).

Note-se que, em termos conceptuais, o direito de participação revelase compatível com as teorias construtivistas do desenvolvimento e da aprendizagem, segundo as quais as crianças são coconstrutoras do seu conhecimento. Em simultâneo, através do exercício do direito de participação, a criança poderá ver satisfeita a necessidade psicológica básica de autonomia (Deci \& Ryan, 2000) e, deste modo, envolver-se mais nos processos de aprendizagem.

Apesar destes potenciais benefícios, estamos, ainda, muito longe de conseguir assegurar o pleno exercício do direito de participação das crianças (Horwath et al., 2011). Existem vários motivos que contribuem para que isso aconteça, nomeadamente a confusão entre os adultos relativamente ao conceito de participação; as barreiras culturais quanto à participação das crianças; a resistência dos adultos à participação das crianças; a falta de vontade de partilhar o poder com as crianças; o predomínio de visões sociais que impedem os adultos de ver as crianças e os jovens como atores sociais e políticos; e a falta de capacidade dos adultos para promover a participação das crianças (Inter-Agency Working Group on Children's Participation, 2008).

Uma vez que a lei consagra o direito de participação das crianças, é necessário perceber como é que este direito é percecionado e implementado em diferentes contextos. Em Portugal, o direito de participação das crianças 
está, de modo implícito, contemplado nas Orientações Curriculares para a Educação Pré-escolar (Ministério da Educação, 1997) e na Lei-Quadro da Educação Pré-escolar (Lei n. ${ }^{\circ}$ 4/97), através da formulação de objetivos relacionados com o desenvolvimento pessoal e social da criança, com vista à educação para uma cidadania democrática. Contudo, existem, ainda, poucas evidências empíricas sobre a forma como o direito de participação das crianças é implementado em contextos de educação de infância, embora várias investigações tenham procurado demonstrar que a qualidade da educação passa pela adoção de uma pedagogia diferenciada, construtivista, e por espaços e práticas participativas protagonizadas com e pelas crianças (e.g., Almeida, 2013; Folque, 2012; Oliveira-Formosinho, 2007).

A Pedagogia da Infância, alinhada com os novos paradigmas da infância, constituiu, desde cedo, um espaço privilegiado para a inauguração de novas formas de pensar e perceber a criança. Assumindo a negociação como instrumento fundamental de participação, Oliveira-Formosinho (2007) identifica duas perspetivas interdependentes da participação em contextos educativos: uma individualista, relacionada com a influência de cada criança no processo de tomada de decisão, e uma comunitária, relacionada com o sentimento de pertença a uma comunidade.

Embora existam diversas lentes para perspetivar as formas e os fins da participação, diferentes modelos pedagógicos para a educação de infância valorizam a pedagogia da participação como meio privilegiado para promover o desenvolvimento e aumentar a confiança das crianças (OliveiraFormosinho, 2007; Oliveira-Formosinho, Kishimoto, \& Pinazza, 2007), como, por exemplo, o modelo HighScope, o modelo Reggio Emilia, ou o Movimento Escola Moderna. Embora com diferentes especificidades, estes modelos assentam numa visão da criança enquanto sujeito ativo: o modelo HighScope defende o papel ativo da criança na sua aprendizagem, enfatizando a sua capacidade para aprender através da realização de atividades por si planeadas e da reflexão sobre as suas ações; o modelo Reggio Emilia defende a igualdade de oportunidades e a partilha de responsabilidades no processo educativo, por toda a comunidade, destacando o atelier como o local para a realização de diversas atividades, através de variadas formas de expressão; o Movimento Escola Moderna é orientado por valores de participação direta, através de estruturas de cooperação educativa (OliveiraFormosinho, 2007). 
Considerando que o jardim de infância constitui um contexto onde as crianças devem exercer o direito de participação e influenciar tudo o que Ihes diga respeito (Sheridan \& Samuelsson, 2001), afigura-se lógico conceptualizar a participação como um indicador da qualidade pedagógica. Consistente com esta conceptualização, existem já evidências de que crianças que frequentam contextos de elevada qualidade relatam mais oportunidades para participar/exercer influência (Sheridan, 2007; Sheridan \& Samuelsson, 2001).

Embora a qualidade pedagógica possa ser perspetivada de variadas formas (Bairrão, 1998; Tobin, 2005), parece ser consensual que as interações educador-criança constituem os mecanismos primordiais de promoção do desenvolvimento e da aprendizagem das crianças. A perspetiva subjacente à operacionalização de qualidade considerada no âmbito deste trabalho propõe três domínios da interação educador-criança, suportados pela teoria e por evidências empíricas: Apoio Emocional (e.g., relações positivas, sensibilidade, consideração pelas perspetivas das crianças), Organização da Sala (e.g., gestão positiva de comportamentos) e Apoio ao Nível da Instrução (e.g., desenvolvimento de conceitos e promoção da linguagem) (ver Hamre, 2014).

Neste estudo, pretende-se desenvolver e avaliar as características psicométricas de uma medida de avaliação da perceção dos educadores de infância acerca do grau de implementação do direito de participação em contexto de jardim de infância. Paralelamente, pretende-se investigar a relação entre a qualidade observada em contexto de jardim de infância e a promoção da participação. Espera-se que o grau de implementação do direito de participação das crianças em contexto de jardim de infância, conforme relatado pelos educadores de infância, esteja positivamente associado à qualidade das interações educador-crianças.

\section{Método}

\section{Participantes}

Participaram neste estudo 168 educadores de infância portugueses, na sua maioria do sexo feminino $(98 \%)$. As idades destes profissionais variaram entre 23 e 63 anos $(M=45.03, D P=9.29)$ e o número de anos de experiência profissional na área da educação de infância variou entre 1 e 36 
$(M=20.71, D P=9.26)$. De acordo com os critérios de seleção definidos, foram recrutados educadores de infância responsáveis por salas destinadas a crianças dos 3 aos 5/6 anos de idade, em estabelecimentos da rede nacional de educação pré-escolar (incluindo jardins de infância públicos, privados com fins lucrativos e privados sem fins lucrativos). A maioria dos educadores exercia funções em entidades públicas $(n=120)$. O setor privado representa cerca de $28.6 \%$ das entidades empregadoras destes profissionais.

O número total de crianças do grupo de cada um destes profissionais variou entre 6 e $27(M=20, D P=4.15)$. A tipologia do grupo de crianças sob a responsabilidade do educador dividiu-se em quatro categorias: grupos mistos $(n=134)$, grupos de crianças de 3 anos $(n=15)$, grupos de crianças de 4 anos $(n=11)$ e grupos de crianças de 5 anos $(n=8)$.

\section{Instrumentos}

Os dados utilizados no âmbito deste estudo foram recolhidos com base em dois instrumentos: (1) um instrumento de autorrelato destinado a captar as perceções dos educadores de infância sobre o grau de implementação do direito de participação das crianças nas suas salas; e (2) um instrumento de observação destinado a captar a qualidade das interações educador-crianças.

\section{Questionário de Avaliação das Perceções dos Educadores de Infância sobre o Direito de Participação das Crianças}

O questionário desenvolvido no âmbito deste estudo tem como objetivo avaliar as perceções dos educadores de infância acerca da implementação do direito de participação das crianças, em contexto de jardim de infância. A construção do questionário baseou-se na revisão da literatura sobre o conceito de participação e práticas de participação (e.g., Hart, 1992) e na análise de conteúdo de documentos nacionais e internacionais relevantes nos domínios da educação de infância, para a identificação de indicadores de participação, incluindo as Orientações Curriculares para a Educação Pré-escolar (Ministério da Educação, 1997), as orientações da National Association for the Education of Young Children (2009), os princípios do Movimento Escola Moderna (Folque, 2012; Niza, 2007) e o Classroom Assessment Scoring System - CLASS (Pianta, La Paro, \& Hamre, 2008). 
recurso aos princípios do Movimento da Escola Moderna (Niza, 2007) para a identificação de indicadores de participação está relacionado com o facto de se tratar de um modelo especificamente desenvolvido e utilizado em jardins de infância portugueses, que se pauta por valores que favorecem a participação das crianças. O CLASS foi analisado na medida em que constitui uma medida internacionalmente reconhecida da qualidade das interações em contextos educativos (ver Hamre, 2014) e inclui uma dimensão que capta especificamente a consideração dos adultos pelas perspetivas das crianças, contemplando indicadores de promoção da responsabilidade e da autonomia, bem como o grau com que os adultos se focam nos interesses e pontos de vista das crianças.

A análise destes documentos permitiu a identificação de 105 unidades de registo consideradas relevantes. Através da análise das unidades de registo, procedeu-se à operacionalização de 38 itens, que contemplavam experiências de participação consideradas relevantes em contexto de jardim de infância como, por exemplo, a possibilidade de a criança poder escolher os materiais a utilizar nas atividades. Os itens foram elaborados de forma a refletir uma formulação positiva, como é possível verificar no seguinte item: "... incluo os interesses e as ideias das crianças nos meus objetivos de trabalho e na minha planificação".

A primeira versão do questionário foi submetida ao escrutínio de peritos nas áreas da psicologia e da sociologia da infância, com investigação nos domínios da qualidade e/ou da participação em contextos de educação de infância, bem como especialistas na construção e validação de instrumentos. Deste modo, cinco peritos procederam à avaliação dos itens quanto à sua relevância, clareza e acessibilidade linguística. Os itens foram medidos numa escala de 4 pontos $(1=$ Nada relevante; $2=$ Pouco relevante; $3=$ Algo Relevante; $4=$ Muito relevante). A mesma escala foi utilizada para a clareza e acessibilidade linguística, substituindo a palavra "relevante" por "claro e acessível". A média das pontuações atribuídas pelos peritos foi elevada nos três aspetos em consideração (Relevância: $M=3.8, D P=0.3$; Clareza: $M=$ 3.6, $D P=0.3$; Acessibilidade: $M=3.4, D P=0.2$ ). Após a análise das respostas de cada perito, e considerando os seus comentários e sugestões, eliminaramse 6 itens e efetuaram-se pequenas alterações em 19, de forma a melhorar a clareza. No final do processo de revisão, os itens foram reordenados com base numa listagem de números aleatórios. 
Posteriormente, foi conduzido um pré-teste, com base na administração do questionário a sete educadores de infância, para aferir se os itens estavam construídos com clareza e para identificar eventuais problemas de formulação. Os respondentes eram todos do sexo feminino e as suas idades variavam entre os 30 e os 49 anos. O questionário não suscitou dúvidas e o tempo de preenchimento variou entre os 10 e os 15 minutos. Considerando a ausência de dúvidas perante os itens apresentados, não foi necessário efetuar novas modificações. A única alteração introduzida no questionário foi a inclusão de informação relativa ao tempo necessário para responder ao mesmo. Pelo facto de não ter sido necessária a alteração de quaisquer itens, estes sete questionários foram incluídos no estudo, fazendo parte da amostra. Os educadores que responderam, na fase do teste piloto, cumpriam os critérios de inclusão para participação no estudo.

A versão final do questionário compreendeu duas secções, sendo a primeira constituída por questões sociodemográficas, incluindo questões sobre os modelos pedagógicos utilizados pelos educadores de infância. A segunda parte era composta por 30 itens destinados a avaliar as perceções dos educadores de infância sobre a implementação do direito de participação das crianças. Os itens foram avaliados numa escala de 5 pontos ( 1 = Nada típico, 2 = Pouco típico, 3 = Moderadamente típico, 4 = Muito típico, $5=$ Extremamente típico)

\section{Classroom Assessment Scoring System - Pre-K}

O CLASS (Pianta et al., 2008) constitui uma medida de observação que avalia a qualidade das interações entre educadores e crianças, tendo sido utilizada, no âmbito deste estudo, para obtenção de dados para estabelecer a validade convergente do instrumento em análise. Esta opção decorreu do facto de o CLASS, ao contrário de outras medidas de avaliação da qualidade, se focalizar exclusivamente na qualidade das interações educador-crianças e de contemplar indicadores consistentes com a promoção do exercício do direito de participação das crianças, incluindo o apoio à expressão das ideias e perspetivas das crianças e a promoção da sua autonomia e iniciativa. $O$ CLASS é composto por dez dimensões, reunidas em três domínios: Apoio Emocional, que se refere ao estabelecimento de relações positivas; Organização da Sala, relativa à gestão de comportamentos e atividades que 
promovem o envolvimento das crianças; e Apoio a Nível da Instrução, que alude às interações que favorecem o desenvolvimento linguístico e cognitivo. A cotação de cada dimensão varia entre 1 (indicador de uma qualidade pobre) e 7 (indicador de uma qualidade elevada).

Neste estudo, o acordo interobservadores, verificado em $30 \%$ das salas observadas $(n=12)$, foi calculado com base no coeficiente de correlação intraclasses (one-way random effects model), tendo variado entre .56 para o Apoio a Nível da Instrução e .66 para o Apoio Emocional. A consistência interna dos referidos domínios variou entre $a=.82$, na Organização da Sala, e $a=.91$ na nota global do CLASS.

\section{Procedimento}

O processo de recolha de dados teve como principal veículo a utilização da plataforma online Qualtrics. O questionário foi divulgado através de redes sociais e de contactos efetuados, por meio eletrónico, junto de educadores de infância ou outros profissionais que desenvolviam a sua atividade junto de educadores de infância. Segundo Gosling, Vazire, Srivastava, e John (2004), a utilização da Internet para aplicação de questionários pode garantir o acesso a amostras de maior dimensão do que aquelas que seriam possíveis de alcançar pelo método tradicional. Além disso, é um método menos dispendioso.

O instrumento foi distribuído através da divulgação de um link com acesso direto ao questionário, precedido das instruções de preenchimento e esclarecendo o respondente que a sua participação era completamente voluntária e anónima, respeitando as exigências éticas, nomeadamente no que diz respeito ao consentimento informado, garantia de proteção dos dados, participação voluntária e informada e respeito pela dignidade humana, conforme o disposto na American Psychological Association (2010) e no Código Deontológico da Ordem dos Psicólogos Portugueses (2011).

Na sua versão online, o questionário foi construído de forma a exigir resposta obrigatória a todos os itens, com a impossibilidade de avançar para a secção seguinte sem o completo preenchimento dos itens. O processo de divulgação foi mantido até ter sido assegurado um mínimo de cinco participantes por item. Além da divulgação do questionário online, 40 
educadores de infância responsáveis por salas observadas com base no CLASS foram convidados a responder ao questionário através do preenchimento em suporte de papel.

A aplicação do CLASS foi efetuada por observadores treinados e certificados, no período da manhã, num dia considerado típico pelo educador. Em cada sala, foram realizados, quatro períodos de observação de, pelo menos, 15 minutos.

\section{Resultados}

Os 30 itens relativos às perceções dos educadores de infância acerca da implementação do direito de participação das crianças em contexto de jardim de infância foram submetidos a uma análise de componentes principais. A verificação dos pressupostos foi aferida previamente, através da adequabilidade dos dados (i.e., assegurando um rácio de, pelo menos, cinco educadores de infância por cada item) e de uma matriz de correlações favorável entre os itens, com vários valores superiores a .30. O valor de Kaiser-Meyer-Olkin foi de .82, o que excede o valor recomendado, e o teste de esfericidade de Bartlett apresentou valor estatisticamente significativo ( $p<$ .001) (Pallant, 2005).

A análise de componentes principais permitiu identificar sete componentes com eigenvalues superiores a 1. No entanto, após a análise do scree plot e o cálculo de uma análise paralela (Patil, Singh, Mishra, \& Donovan, 2007), constatou-se que uma solução de dois componentes era adequada. No decurso da análise de componentes principais e para interpretar melhor os componentes, foi realizada uma rotação oblíqua Oblimin, que convergiu após cerca de 60 iterações. De forma sequencial, foram eliminados quatro itens (...as crianças escolhem os parceiros de brincadeira; ... as crianças são informadas acerca das atividades planeadas por mim; ... as crianças escolhem os materiais utilizados nas atividades; ...defino o plano de atividades em conjunto com as crianças) que contribuíam de forma idêntica para ambos os componentes ou que não contribuíam para nenhum componente, até à obtenção da solução final, apresentada no Quadro 1. 


\section{Quadro 1 - Resultados da análise de componentes principais dos itens relativos às perceções dos educadores de infância sobre a implementação do direito de participação das crianças $(\mathbf{N}=168)$}

\begin{tabular}{|c|c|c|c|c|c|}
\hline \multirow[b]{3}{*}{ Na minha sala... } & \multirow[t]{3}{*}{ Comunalidades } & \multirow{2}{*}{\multicolumn{2}{|c|}{$\begin{array}{c}\text { Matriz de } \\
\text { padrão } \\
\text { Componente }\end{array}$}} & \multicolumn{2}{|c|}{$\begin{array}{l}\text { Matriz de } \\
\text { estrutura }\end{array}$} \\
\hline & & & & \multicolumn{2}{|c|}{ Componente } \\
\hline & & 1 & 2 & 1 & 2 \\
\hline $\begin{array}{l}\text {..estimulo as crianças a expressar publicamente a sua opinião } \\
\text { (e.g., debates). }\end{array}$ & 0.51 & .75 & .18 & .69 & -.06 \\
\hline $\begin{array}{l}\text {...proporciono oportunidades diárias para as crianças } \\
\text { expressarem as suas ideias e opiniões. }\end{array}$ & 0.52 & .74 & .09 & .72 & -.15 \\
\hline $\begin{array}{l}\text {...proporciono oportunidades diárias para as crianças } \\
\text { partilharem as suas vivências pessoais nas atividades de grupo. }\end{array}$ & 0.49 & .71 & .03 & .70 & -.20 \\
\hline $\begin{array}{l}\text {...as crianças participam na definição das tarefas inerentes ao } \\
\text { funcionamento da sala. }\end{array}$ & 0.48 & .64 & -.13 & .68 & -.34 \\
\hline $\begin{array}{l}\text {...as crianças fazem propostas de atividades e brincadeiras aos } \\
\text { adultos. }\end{array}$ & 0.40 & .61 & -.07 & .63 & -.27 \\
\hline $\begin{array}{l}\text {...as regras indispensáveis à vida em comum são elaboradas em } \\
\text { conjunto com as crianças. }\end{array}$ & 0.34 & .58 & -.01 & .58 & -.19 \\
\hline $\begin{array}{l}\text {...as crianças participam nos momentos de avaliação do trabalho } \\
\text { desenvolvido. }\end{array}$ & 0.45 & .58 & -.20 & .64 & -.38 \\
\hline $\begin{array}{l}\text {...as situações problemáticas são debatidas em grupo, tentando } \\
\text { que as crianças encontrem as suas próprias soluções. }\end{array}$ & 0.34 & .57 & -.03 & .58 & -.22 \\
\hline $\begin{array}{l}\text {...modifico os meus planos para desenvolver atividades } \\
\text { relacionadas com os interesses momentâneos das crianças. }\end{array}$ & 0.30 & .57 & .11 & .53 & -.07 \\
\hline $\begin{array}{l}\ldots \text { incluo os interesses e as ideias das crianças nos meus } \\
\text { objetivos de trabalho e na minha planificação. }\end{array}$ & 0.32 & .56 & -.02 & .57 & -.20 \\
\hline $\begin{array}{l}\text {...as crianças são responsáveis por tarefas do dia-a-dia, } \\
\text { necessárias à vida coletiva (e.g., alimentar um animal de } \\
\text { estimação, marcar as presenças, etc.). }\end{array}$ & 0.25 & .51 & .04 & .50 & -.12 \\
\hline $\begin{array}{l}\text {... as crianças têm liberdade de movimentos e podem decidir } \\
\text { onde brincar/trabalhar. }\end{array}$ & 0.31 & .51 & -.12 & .55 & -.28 \\
\hline $\begin{array}{l}\text {...os trabalhos e materiais expostos estão colocados ao nível e } \\
\text { ao alcance das crianças. }\end{array}$ & 0.22 & .47 & .00 & .47 & -.15 \\
\hline $\begin{array}{l}\text {...as crianças são responsáveis pelo registo das atividades que } \\
\text { escolhem. }\end{array}$ & 0.23 & .41 & -.16 & .46 & -.28 \\
\hline $\begin{array}{l}\text {...a maior parte dos materiais expostos foi elaborada pelas } \\
\text { crianças. }\end{array}$ & 0.21 & .38 & -.16 & .43 & -.28 \\
\hline $\begin{array}{l}\text {...sou eu que defino a organização das áreas e materiais em } \\
\text { função das características do espaço e dos objetivos que me } \\
\text { propus atingir com o grupo. }\end{array}$ & 0.55 & .07 & .76 & -.17 & .74 \\
\hline $\begin{array}{l}\text {...sou eu que defino o plano de atividades de forma a assegurar } \\
\text { o cumprimento dos objetivos que me propus atingir com o grupo. }\end{array}$ & 0.52 & -.05 & .71 & -.28 & .72 \\
\hline $\begin{array}{l}\text {...sou eu que defino as atividades e brincadeiras, de acordo com } \\
\text { os materiais e espaço disponíveis. }\end{array}$ & 0.48 & -.09 & .66 & -.30 & .69 \\
\hline ...sou eu que defino as regras que as crianças têm que cumprir. & 0.41 & -.05 & .62 & -.24 & .64 \\
\hline $\begin{array}{l}\text {..todas as crianças fazem os mesmos trabalhos, com os } \\
\text { mesmos materiais. }\end{array}$ & 0.39 & -.06 & .60 & -.25 & .62 \\
\hline $\begin{array}{l}\text {...são as crianças que escolhem os temas para os projetos a } \\
\text { desenvolver. (Invertido) }\end{array}$ & 0.48 & -.24 & .58 & -.42 & .66 \\
\hline $\begin{array}{l}\text {...as crianças sabem que há momentos para trabalhar e } \\
\text { momentos para conversar. }\end{array}$ & 0.30 & .28 & .57 & .10 & .48 \\
\hline $\begin{array}{l}\text {...o horário é determinado por mim ou pela coordenação e as } \\
\text { crianças sabem que têm de o cumprir. }\end{array}$ & 0.22 & .05 & .48 & -.10 & .46 \\
\hline $\begin{array}{l}\text {...sou eu que escolho os companheiros de brincadeira das } \\
\text { crianças de modo a evitar problemas e a atingir determinados } \\
\text { objetivos. }\end{array}$ & 0.19 & -.05 & .41 & -.18 & .43 \\
\hline $\begin{array}{l}\text {...as crianças participam na tomada de decisões relativas à } \\
\text { organização/dinâmica do jardim de infância (e.g., horários; } \\
\text { regras; passeios). (Invertido) }\end{array}$ & 0.21 & -.20 & .35 & -.310 & .41 \\
\hline $\begin{array}{l}\text {...as crianças decidem onde sentar-se, durante as refeições. } \\
\text { (Invertido) }\end{array}$ & 0.14 & -.14 & .31 & -.235 & .35 \\
\hline
\end{tabular}


O primeiro componente agrega itens que remetem para a liberdade de expressão e para aspetos relacionados com a definição de regras e responsabilidade por tarefas diárias, pelo que se adotou o nome Expressão e Responsabilidade das Crianças ( $a=.85$ ). Os itens que constituem o segundo componente configuram essencialmente questões relacionadas com a iniciativa da decisão pelo adulto, pelo que este componente foi designado como Tomada de Decisão pelo Adulto ( $\mathrm{a}=.78$ ). Os dois componentes explicam $35.62 \%$ da variância total, sendo que o primeiro componente explica $25.29 \%$ e o segundo componente explica $10.33 \%$ da variância.

No caso da subescala Tomada de Decisão pelo Adulto, foram identificados três itens que contribuíam negativamente para o componente e que, por isso, foram invertidos na solução final. As estatísticas descritivas das componentes ou subescalas relativas às perceções dos educadores sobre a implementação do direito de participação das crianças figuram no Quadro 2. A média das respostas à subescala Tomada de Decisão pelo Adulto indica que, de um modo geral, o peso dos adultos na tomada de decisão é moderado. As perceções sobre a Expressão e Responsabilidade das Crianças apresentaram médias elevadas, indicativas que os educadores participantes consideram que as crianças têm liberdade de expressão e podem ser responsáveis por tarefas diárias.

\section{Quadro 2 - Médias, desvios-padrão e amplitude dos dados das subescalas relativas às perceções dos educadores sobre a implementação do direito de participação das crianças $(\mathrm{N}=168)$}

\begin{tabular}{lcccc}
\hline & $M$ & $D P$ & Mín. & Máx. \\
\hline Expressão e Responsabilidade das Crianças & 4.38 & 0.40 & 3.07 & 5.00 \\
Tomada de Decisão pelo Adulto & 2.89 & 0.56 & 1.55 & 4.27 \\
\hline
\end{tabular}

A título exploratório, no sentido de informar acerca da adequação de eventuais análises com base numa escala única de perceções dos educadores, calculou-se o Alfa de Cronbach para a totalidade dos itens. Assim, quando considerados os 30 itens relativos às perceções dos educadores de infância sobre a implementação do direito de participação das 
crianças, em contexto de jardim de infância, obteve-se $\alpha=.70$. Após a inversão de oito itens que apresentavam correlações negativas com o total da escala e de um item formulado em sentido inverso, do ponto de vista conceptual, obteve-se um coeficiente Alfa de Cronbach de .88 .

Não foram apuradas diferenças estatisticamente significativas nas perceções dos educadores de infância relativamente à Tomada de Decisão pelo Adulto, em função da idade $\left(t_{(165)}=0.788, p=.432\right)$ e dos anos de experiência em educação de infância $\left(t_{(165)}=1.430, p=.155\right)$. Do mesmo modo, não se verificaram diferenças estatisticamente significativas entre os educadores que trabalhavam no setor público ou no setor privado com e sem fins lucrativos relativamente às perceções sobre a Tomada de Decisão pelo Adulto $\left(F_{(2 ; 165)}=1.474, p=.232\right)$.

Os resultados de uma one-way Anova indicaram que existiam diferenças estatisticamente significativas nas perceções sobre a Tomada de Decisão pelo Adulto, em função do tipo de grupo das crianças $\left(F_{(3 ; 164)}=\right.$ $4.771, p=.003$ ). Com base no teste de comparações múltiplas Scheffe, foram apuradas diferenças estatisticamente significativas entre os educadores que tinham grupos de crianças de quatro anos e os educadores que tinham sob a sua responsabilidade grupos mistos $(p=.029)$. Os educadores com grupos mistos obtiveram uma média de respostas inferior $(M=2.82)$, face aos educadores com grupos de crianças de quatro anos $(M=3.34)$. Estima-se, com um nível de confiança de 95\%, que o verdadeiro valor médio das respostas nesta subescala se situa entre 2.73 e 2.91 para os educadores com grupos de crianças mistos. Para o caso dos educadores com grupos de crianças de quatro anos, esses valores situam-se entre 2.88 e 3.81 .

No que diz respeito às perceções dos educadores sobre a Expressão e Responsabilidade das Crianças, foram efetuados testes não paramétricos, devido à violação do pressuposto de normalidade da distribuição. À semelhança dos resultados obtidos para as perceções sobre a Tomada de Decisão pelo Adulto, não foram encontradas diferenças estatisticamente significativas em função da idade dos educadores $(U=3111.500, z=-1.053$, $p=.292)$ e da sua experiência em educação de infância $(U=3210.500, z=-$ $0.756, p=.450)$. 
Contudo, os resultados do teste de Kruskal-Wallis revelaram a existência de diferenças estatisticamente significativas nas perceções dos educadores em relação à Expressão e Responsabilidade das Crianças em função do tipo de instituição em que trabalhavam $\left(H_{(2)}=7.108, p=.029\right)$. Com base no teste de comparação múltipla Scheffe, apuraram-se diferenças ( $p=$ .030) entre o setor público $(M=4.42)$ e o setor privado com fins lucrativos ( $M$ = 4.17). Considerando um nível de confiança de 95\%, estima-se que o verdadeiro valor médio das respostas à subescala se situa entre 4.35 e 4.49 para o setor público e entre 3.97 e 4.36 para o setor privado com fins lucrativos, sendo a variabilidade dos dados superior neste último grupo.

No que diz respeito ao tipo de grupo sob a responsabilidade dos educadores, verificou-se a existência de diferenças estatisticamente significativas $\left(H_{(3)}=8.671, p=.034\right)$. Contudo, após o cálculo do teste de comparação múltipla Scheffe, não foram encontradas diferenças entre pares de grupos.

De modo a verificar a direção e magnitude das associações entre os dois componentes obtidos neste estudo e entre estes e determinadas variáveis sociodemográficas, foram calculados coeficientes de correlação de Spearman, uma vez que a maioria das variáveis em análise apresentava distribuição não normal. No Quadro 3, é possível verificar a existência de uma associação positiva, estatisticamente significativa, entre as perceções sobre a Expressão e Responsabilidade das Crianças e o número total de crianças no grupo do educador, bem como uma associação negativa, estatisticamente significativa, entre as perceções de Tomada de Decisão pelo Adulto e as habilitações académicas do educador de infância. Finalmente, apurou-se uma associação negativa moderada, estatisticamente significativa, entre as perceções de Tomada de Decisão pelo Adulto e as perceções relativas à Expressão e Responsabilidade das Crianças. 
Quadro 3 - Coeficientes de correlação de Spearman entre as perceções dos educadores sobre a implementação do direito de participação das crianças e variáveis sociodemográficas $(\mathrm{N}=168)$

\begin{tabular}{lcccc}
\hline & 1 & 2 & 3 & 4 \\
\hline 1. Habilitações académicas do educador & --- & & & \\
2. Número total de crianças no grupo do educador & -.05 & --- & & \\
3. Anos de serviço como educador de infância & $-.21^{* *}$ & .09 & --- & \\
4. Expressão e responsabilidade das crianças & .05 & .16 & .02 & --- \\
5. Tomada de decisão pelo adulto & -.26 & -.07 & -.02 & $-.40^{* *}$ \\
\hline${ }^{*} p<.05 .{ }^{* *} p<.01$. & & & &
\end{tabular}

No Quadro 4, são apresentadas as correlações entre as subescalas relativas às perceções dos educadores sobre participação e os resultados relativos à qualidade observada nas salas, avaliada com base no CLASS. Foram considerados apenas os dados dos educadores que responderam ao inquérito e foram, simultaneamente, observados em contexto de sala $(N=40)$. Recorreu-se ao coeficiente de correlação de Spearman, uma vez que a maioria das variáveis apresentava distribuição não normal. Apenas foram apuradas associações estatisticamente significativas, negativas e de intensidade moderada, entre os três domínios e a pontuação total do CLASS e a subescala relativa às perceções de Tomada de Decisão pelo Adulto.

Quadro 4 - Coeficientes de correlação de Spearman entre as perceções dos educadores sobre a implementação do direito de participação das crianças e a qualidade observada com o CLASS $(\mathrm{N}=40)$

\begin{tabular}{lcccc}
\hline & $\begin{array}{c}\text { Apoio } \\
\text { Emocional }\end{array}$ & $\begin{array}{c}\text { Organização } \\
\text { da Sala }\end{array}$ & $\begin{array}{c}\text { Apoio à } \\
\text { Instrução }\end{array}$ & $\begin{array}{c}\text { CLASS } \\
\text { (pontuação } \\
\text { total) }\end{array}$ \\
\hline $\begin{array}{l}\text { Expressão } e \\
\begin{array}{l}\text { Responsabilidade das } \\
\text { Crianças }\end{array}\end{array}$ & .26 & .22 & .19 & .28 \\
$\begin{array}{l}\text { Tomada de Decisão pelo } \\
\text { Adulto }\end{array}$ & $-.47^{* *}$ & $-.34^{*}$ & $-.51^{* \star}$ & $-.49^{* *}$ \\
\hline${ }^{*} p<.05 .^{* \star} p<.01$ & & &
\end{tabular}




\section{Discussão}

Com este trabalho, pretendeu-se desenvolver e avaliar as características psicométricas de uma medida de avaliação das perceções dos educadores de infância acerca do grau de implementação do direito de participação das crianças, em contexto de jardim de infância. Deste modo, procedeu-se à verificação da validade de constructo através de uma análise de componentes principais. Esta análise permitiu identificar dois componentes relativos às perceções dos educadores sobre a implementação do direito de participação das crianças, designadamente Expressão e Responsabilidade das Crianças e Tomada de Decisão pelo Adulto. A emergência destes dois componentes traduz algumas dimensões do conceito de participação. No primeiro componente, Expressão e Responsabilidade das Crianças, encontravam-se presentes itens que sugeriam liberdade de expressão e envolvimento na definição de regras e nas tarefas diárias. A liberdade de expressão é uma das dimensões enunciadas nos artigos $12 .^{\circ}$ e $13 .^{\circ}$ da CDC (1989). A possibilidade de a criança participar na definição de regras e nas tarefas no dia-a-dia do jardim de infância, ou seja, a possibilidade de influenciar as decisões sobre as atividades diárias, bem como o envolvimento nas mesmas, está presente nos níveis de participação propostos por Hart (1992), nomeadamente no que diz respeito à partilha de decisões com as crianças e ao estímulo para que as crianças expressem as suas opiniões, assegurando que estas sejam ouvidas. O envolvimento numa atividade, enquanto dimensão de participação, está também presente nos itens que integram este componente (Almqvist, Uys, \& Sandberg, 2007; Sandberg \& Eriksson, 2010).

O segundo componente, Tomada de Decisão pelo Adulto, inclui itens que remetem, de algum modo, para limitações à participação da criança, por não contemplarem a criança como parceiro na tomada de decisão ou como decisor. Os itens descrevem situações em que é o adulto que decide os aspetos relativos às atividades diárias em contexto de jardim de infância, incluindo aspetos relacionados com atividades de lazer e com o cumprimento de objetivos pedagógicos. Este componente reflete, essencialmente, o papel do adulto, conforme referem Reddy e Ratna (2002). A criança não é convidada a fazer parte do processo de tomada de decisão; ou seja, os adultos prosseguem os seus objetivos pedagógicos, de acordo com as características 
do grupo de crianças e regras institucionais e não partilham a tomada de decisão.

A verificação da fidelidade dos dados permitiu verificar que ambos os componentes - Expressão e Responsabilidade das Crianças e Tomada de Decisão pelo Adulto - revelaram boa consistência interna, podendo ser utilizadas como variáveis em análises subsequentes.

A média de respostas ao componente Expressão e Responsabilidade das Crianças foi elevada, demonstrando que, de acordo com os relatos dos educadores de infância, a liberdade de expressão e a participação das crianças nas atividades diárias eram muito típicas dos contextos de jardim de infância. Por outro lado, as respostas ao componente Tomada de Decisão pelo Adulto revelaram que, de acordo com o relato dos educadores, o papel preponderante do adulto na tomada de decisão era moderadamente típico. Estes dados parecem ser consistentes com as Orientações Curriculares para a Educação Pré-escolar (Ministério da Educação, 1997), na medida em que parecem refletir a valorização da promoção da participação das crianças. São, também, globalmente consistentes com a investigação prévia. Por exemplo, Samuelsson, Sheridan, e Williams (2006) concluíram que, em educação de infância, é comum considerar-se o direito de as crianças serem livres de expressar a sua opinião sobre o que as rodeia. Paralelamente, Sheridan e Samuelsson (2001) reuniram evidências de que as crianças podem decidir sobre que jogos e atividades realizar, apesar de terem pouca influência relativamente a aspetos relacionados com a organização global do contexto educativo.

As perceções dos educadores relativamente à Expressão $e$ Responsabilidade das Crianças e à Tomada de Decisão pelo Adulto não variaram em função de variáveis sociodemográficas como a idade e a experiência em educação de infância. Em investigações anteriores, foram encontradas diferenças nas práticas dos educadores em função da sua formação e especialização profissional (Wen, Elicker, \& McMullen, 2011).

Neste estudo, verificaram-se diferenças entre os educadores relativamente às perceções sobre a Expressão e Responsabilidade das Crianças, sugerindo que, de acordo com o relato dos educadores, a implementação do direito de participação das crianças ao nível da liberdade de expressão e participação nas atividades diárias é maior no setor público do 
que no setor privado com fins lucrativos. A ocorrência destas diferenças poderá estar relacionada com a natureza das organizações e com diferenças nas expectativas e pressões das famílias, que poderão influenciar os objetivos e práticas dos educadores (Hyson, Hirsh-Pasek, \& Rescorla, 1990; Stipek \& Byler,1997).

Paralelamente, verificaram-se diferenças nas perceções dos educadores relativamente à Tomada de Decisão pelo Adulto em função da composição etária do grupo de crianças, sugerindo que, de acordo com o relato dos educadores, a preponderância do papel do adulto na tomada de decisão é maior em grupos homogéneos de crianças de quatro anos. A diferença das perceções dos educadores em função da composição etária do grupo pode estar relacionada com a forma como encaram o desenvolvimento da criança e com a forma de as incluir no processo de tomada de decisão. Stipek e Byler (1997) reforçam esta ideia, sugerindo que o quadro referencial teórico sobre o desenvolvimento cognitivo da criança, os objetivos dos educadores e a idade da criança (Tarman, 2012) condicionam as suas perceções/crenças. É possível sugerir, também, como hipótese explicativa, que nos grupos mistos o educador recorra às crianças mais velhas como recurso, partilhando com o grupo a tomada de decisão. Por outro lado, em grupos homogéneos de crianças mais novas o educador poderá sentir necessidade de assumir, em maior grau, a responsabilidade pelas decisões.

No que diz respeito à Tomada de Decisão pelo Adulto, verificaram-se associações negativas com as habilitações académicas dos educadores, sugerindo que aquela é menos típica em salas de educadores de infância com níveis mais elevados de educação formal. A relação entre níveis académicos mais elevados e práticas pedagógicas promotoras da participação é convergente com os resultados do estudo de Wen et al. (2011), segundo os quais os educadores que foram submetidos a formação específica na área da educação de infância utilizam frequentemente abordagens pedagógicas mais apropriadas ao desenvolvimento da criança e ao seu envolvimento no processo de aprendizagem. É expectável que os educadores com habilitações académicas mais elevadas tenham maior acesso e exposição a práticas educativas promotoras de participação.

A relação entre o número total de crianças do grupo e as perceções dos educadores sobre a Expressão e Responsabilidade das Crianças é 
positiva, o que significa que, quanto maior é o número de crianças no grupo, maior é a participação das crianças, de acordo com o relato dos educadores. Estes resultados podem indicar que os educadores, perante grupos maiores de crianças, poderão sentir a necessidade de implicar mais as crianças nas tarefas diárias. Esta abordagem permite uma maior integração da diversidade de experiências individuais das crianças e, assim, facilita o treino de processo democrático, conforme é sugerido nas Orientações Curriculares para a Educação Pré-escolar (Ministério da Educação, 1997).

A relação negativa entre as subescalas de Tomada de Decisão pelo Adulto e de Expressão e Responsabilidade das Crianças era expectável devido ao facto de cada escala medir dimensões opostas do direito de participação. A primeira subescala reflete a desvalorização da participação da criança, que poderá estar implicitamente associada à confusão entre os adultos acerca do conceito de participação, à resistência relativamente à participação da criança e à falta de vontade em partilhar o poder com as crianças (Inter-Agency Working Group on Children's Participation, 2008). A segunda subescala compreende itens promotores da participação da criança como é proposto, por exemplo, nos níveis de participação descritos por Kirby e colaboradores (2003), em que os pontos de vista das crianças são tidos em consideração e estas são envolvidas no processo de tomada de decisão. Estão também presentes as dimensões de partilha do poder, a necessidade de ouvir as vozes das crianças (Sinclair, 2004) e o envolvimento em atividades (Sandberg \& Eriksson, 2010), como brincar e fazer amigos (Almqvist et al., 2007).

Apenas a subescala Tomada de Decisão pelo Adulto apresentou correlações negativas e de intensidade moderada com os vários domínios do CLASS. Esta associação parece indicar que os educadores que relatam mais práticas baseadas na Tomada de Decisão pelo Adulto manifestam, em contexto de sala, menos apoio emocional e práticas de menor qualidade ao nível da gestão de comportamentos e da promoção do desenvolvimento cognitivo e da linguagem. Estas correlações poderão constituir indicadores de validade convergente da subescala de Tomada de Decisão pelo Adulto. Assim, considera-se que a hipótese formulada neste estudo segundo a qual o grau de implementação do direito de participação das crianças em contexto de jardim de infância estaria associado à qualidade das interações educadorcriança foi parcialmente confirmada. Estes resultados parecem convergir com 
estudos anteriores que referem a relação entre qualidade e participação, sugerindo que a participação das crianças e o seu envolvimento no processo de tomada de decisão são indicadores da qualidade dos contextos educativos (Sheridan, 2007; Sheridan \& Samuelsson, 2001). Aliás, a qualidade é definida, por alguns autores (e.g., Balageur, Mestres, \& Penn, 1991), como tendo em consideração os direitos da criança, nomeadamente a liberdade de expressão, o que parece revelador da importância da participação. Esta ideia é, também, visível nas propostas de Pianta e Hamre (2009) relativamente à avaliação da qualidade das interações educador/ professor-crianças, fundamentadas nas teorias da vinculação e da autodeterminação.

Considerando os aspetos metodológicos relativos à recolha de dados, alguns autores consideram que a utilização da Internet para a recolha de dados pode gerar respostas repetidas e não permitir diversidade demográfica (ver Gosling et al., 2004), pelo que este estudo poderia estar, de alguma forma, limitado a este nível. Contudo, Gosling e colaboradores verificaram que os dados recolhidos através da Internet são tão consistentes como os dados recolhidos através de métodos tradicionais. Nesse sentido, consideramos que a recolha de dados, através da Internet foi uma opção oportuna e prática, tendo em conta o tempo e recursos disponíveis.

A amostra utilizada neste trabalho poderá não ser representativa da população de educadores de infância, pelo que a generalização dos resultados obtidos, para a população, exige, necessariamente, cautela. Assim, em investigações futuras, a aplicação do instrumento em estudo a uma amostra superior, bem como o aumento do número de salas observadas com recurso ao CLASS (ou outra medida de observação da qualidade de processo), será vantajosa.

Uma potencial limitação adicional deste trabalho remete para a necessidade de inclusão e operacionalização de outros conceitos/dimensões associados à implementação do direito de participação das crianças em contexto de jardim de infância, incluindo os obstáculos à participação.

No que diz respeito à fidelidade da medida em estudo, não foi possível determinar a sua estabilidade em termos temporais, através da aplicação teste-reteste (Coutinho, 2014). Seria importante realizar um estudo de carácter longitudinal que permitisse aferir o comportamento dos dados em, pelo menos, dois momentos distintos, a fim de obter índices adicionais acerca 
da fidelidade. Finalmente, os resultados relativos à validade convergente da medida em estudo poderão não ser suficientemente robustos, uma vez que apenas uma das subescalas do Questionário de Avaliação das Perceções dos Educadores de Infância sobre o Direito de Participação das Criança estava associada aos domínios do CLASS. No entanto, é de realçar que a análise da associação entre as duas medidas constitui uma mais-valia, que contribuiu para o rigor científico do trabalho desenvolvido.

Apesar das limitações referidas, o Questionário de Avaliação das Perceções dos Educadores de Infância sobre o Direito de Participação das Criança constitui uma medida fundamentada em pressupostos conceptuais, que reúne características de fidelidade e validade. De um modo geral, foi possível confirmar a consistência interna das duas subescalas. A submissão do questionário ao escrutínio de peritos permitiu validar os itens quanto à sua clareza e relevância e a realização do pré-teste foi, também, uma forma de confirmar a clareza dos itens, junto dos participantes. A análise de componentes principais proporcionou dados relativos à validade de constructo, tendo sido possível captar diferenças entre educadores em função do tipo de instituição em que desempenhavam funções e do tipo de grupo que tinham sob a sua responsabilidade. Estes aspetos, interligados, permitiram fornecer informação quanto às características psicométricas da medida por nós elaborada.

Em suma, este estudo contribuiu para a criação e validação de um novo instrumento que permite avaliar as perceções dos educadores de infância sobre o grau de implementação do direito de participação das crianças em contexto de jardim de infância. Colmatando uma lacuna da investigação neste âmbito, apresenta-se à comunidade científica e profissional um recurso novo, com potencial para contribuir para a investigação acerca da participação das crianças em contexto de jardim de infância.

\section{Nota}

1 Este trabalho baseia-se na tese de mestrado da primeira autora e enquadrou-se no âmbito do projeto de investigação "Promover as relações entre pares: As ideias e as práticas de educadores de infância", tendo sido parcialmente financiado por Fundos Nacionais, via FCT - Fundação para a Ciência e a Tecnologia (PTDC/CPECED/117476/2010). 


\section{Referências}

Almeida, M. B. (2013). Direitos de participação das crianças: Estudo de caso num jardim de infância em contexto do Movimento da Escola Moderna (Tese de mestrado não publicada). Escola Superior de Educação de Lisboa, Instituto Politécnico de Lisboa, Portugal.

Almqvist, L., Uys, C. J. E., \& Sandberg, A. (2007). The concepts of participation, engagement and flow: A matter of creating optimal play experiences. South African Journal of Occupational Therapy, 37(7), 6-13. Retirado de: http://repository.up.ac.za/bitstream/handle/2263/6233/Almqvist_Concepts\% 282007\%29.pdf?sequence=1

American Psychological Association (2010). Ethical principles of psychologists and code of conduct. Washington, DC: APA.

Bairrão, J. (1998). O que é a qualidade em educação pré-escolar? Alguns resultados acerca da qualidade da educação pré-escolar em Portugal. In DEB/GEDEP (Ed.), Qualidade e projecto na educação pré-escolar (pp. 43-88). Lisboa: Departamento da Educação Básica/Gabinete para a Expansão e Desenvolvimento da Educação Pré-Escolar do Ministério da Educação.

Balageur, I., Mestres, J., \& Penn, H. (1991). Quality in services for young children: A discussion paper [EU Commission - Working Document]. Retirado de: http://aei.pitt.edu/39329/1/A3833.pdf

Coutinho, C. P. (2014). Metodologia de investigação em Ciências Sociais e Humanas: Teoria e prática (2. ${ }^{a}$ ed.). Coimbra: Almedina.

Deci, E., \& Ryan, R. (2000). Self-determination theory and the facilitation of intrinsic motivation, social development, and well-being. American Psychologist, 55(1), 68-78. doi: 10.1037110003-066X.55.1.68

Folque, A. (2012). O aprender a aprender no pré-escolar: O modelo pedagógico do Movimento da Escola Moderna. Lisboa: Fundação Calouste Gulbenkian/Fundação para a Ciência e Tecnologia.

Gesellschaft für Technische Zusammenarbeit [GTZ] (2010). Toolkit "Get youth on board!". Youth participation. Eschborn: Deutsche Gesellschaft für Technische Zusammenarbeit.

Gosling, S. D., Vazire, S., Srivastava, S., \& John., P. O. (2004). Should we trust webbased studies? A comparative analysis of six preconceptions about Internet questionnaires. American Psychologist, 59(2), 93-104. doi: 10.1037/0003066X.59.2.93

Hamre, B. K. (2014). Teachers' daily interactions with children: An essential ingredient in effective early childhood programs. Child Development Perspectives, 8(4), 223230. doi: $10.1111 /$ cdep. 12090

Hart, R. (1992). Children's participation: From tokenism to citizenship. Florence, Italy: UNICEF International Child Development Centre.

Horwath, J., Hodgkiss, D., Kalyva, E., \& Spyrou, S. (2011). You respond. Promoting effective project participation by young people who have experienced violence. A guide to good practice through training and development. Retirado de: http://c.ymcdn.com/sites/www.ispcan.org/resource/resmgr/you_respond_ booklet._for_dow.pdf. 
Hyson, M., Hirsh-Pasek, K., \& Rescorla, L. (1990). The classroom practices inventory: An observation instrument based on NAEYC's guidelines for developmentally appropriate practices for 4- and 5-year-old children. Early Childhood Research Quarterly, 5, 475-494. doi:10.1016/0885-2006(90)90015-S

Inter-Agency Working Group on Children's Participation (2008). Children as active citizens: A policy and programme guide. Bangkok: Inter-Agency Working Group on Children's Participation. Retirado de: http://www.unicef.org/eapro/Children_ as_Active_Citizens_A4_book.pdf

Kirby, P., \& Bryson, S. (2002). Measuring the magic? Evaluating and researching young people's participation in public decision-making. London: Carnegie Young People Initiative.

Kirby, P., Lanyon, C., Cronin, K., \& Sinclair, R. (2003). Building a culture of participation: Involving children and young people in policy, service planning, delivery and evaluation. Nottingham: Department for Education and Skills Publications.

Lansdown, G. (2001). Promoting children's participation in democratic decision-making. Itália: UNICEF Innocenti Research Centre.

Ministério da Educação (1997). Orientações curriculares para a educação pré-escolar. Lisboa: Autor.

National Association for the Education of Young Children (2009). Developmentally appropriate practice in early childhood programs serving children from birth through age 8: A position statement of the National Association for the Education of the Young Children. Washington, DC: Author.

Niza, S. (2007). O modelo curricular de educação pré-escolar da Escola Moderna Portuguesa. In J. Oliveira-Formosinho (Org.), D. Lino, \& S. Niza, Modelos curriculares para a educação de infância: Construindo uma práxis de participação (3. ${ }^{a}$ ed., pp. 123-142). Porto: Porto Editora.

Oliveira-Formosinho, J. (2007). Pedagogia(s) da infância: Reconstruindo uma práxis de participação. In J. Oliveira-Formosinho (Org.), D. Lino, \& S. Niza, Modelos curriculares para a educação de infância: Construindo uma práxis de participação ( $3^{\mathrm{a}}$ ed., pp. 13-42). Porto: Porto Editora.

Oliveira-Formosinho, J., Kishimoto, T., \& Pinazza, M. (Orgs.). (2007). Pedagogia(s) da infância: Dialogando com o passado, construindo o futuro. Porto Alegre: Artmed Editora.

Ordem dos Psicólogos Portugueses (2011). Código Deontológico da Ordem dos Psicólogos Portugueses. Diário da República $2^{\mathrm{a}}$ série, 78, 17931-17936.

Pallant, J. (2005). SPSS Survival Manual (2. ${ }^{a}$ ed.). Australia: Allen \& Unwin.

Patil, V. H., Singh, S. N., Mishra, S., \& Donavan, D. T. (2007). Parallel analysis engine to aid determining number of factors to retain [Computer software]. Disponivel em: http://smishra.faculty.ku.edu/parallelengine.htm.

Pianta, R. C., \& Hamre, B. K. (2009). Conceptualization, measurement, and improvement of classroom processes: Standardized observation can leverage capacity. Educational Researcher, 38(2), 109-119. doi: 10.3102/0013189X093 32374 
Pianta, R. C., La Paro, K. M., \& Hamre, B. K. (2008). Classroom Assessment Scoring System Manual: Pre-K. Baltimore, MD: Brookes.

Prout, A., \& James, A. (1997). A new paradigm for the sociology of childhood? Provenance, promise and problems. In A. James \& A. Prout (Eds.), Constructing and reconstructing childhood (pp. 7-31). London: Falmer Press.

Reddy, N., \& Ratna, K. (2002). A journey in children's participation. India: Concerned for working children. Retirado de: http://www.pronats.de/assets/Uploads/ reddyratna-a-journey-in-childrens-participation.pdf.

Samuelsson, I. P., Sheridan, S., \& Williams, P. (2006). Five preschool curricula: Comparative perspective. International Journal of Early Childhood, 38(1), 11-30. doi: 10.1007/BF03165975

Sandberg, A., \& Eriksson, A. (2010). Children's participation in preschool: On the conditions of adults? Preschool staffs concepts of children's participation in preschool everyday life. Early Child Development and Care, 280(5), 619-631. doi:10.1080/03004430802181759

Sarmento, M. J., Fernandes, N., \& Tomás, C. (2006). Participação social e cidadania ativa das crianças. In D. Rodrigues (Ed.), Inclusão e educação: Doze olhares sobre a educação inclusiva (pp. 141-159). São Paulo: Summus Editorial.

Sheridan, S. (2007). Dimensions of pedagogical quality in preschool. International Journal of Early Years Education, 15(2), 197-217. doi: 10.1080/0966976070 1289151

Sheridan, S., \& Samuelsson, I. P. (2001). Children's conceptions of participation and influence in preschool: A perspective on pedagogical quality. Contemporary Issues in Early Childhood, 2(2), 169-194. doi:10.2304/ciec.2001.2.2.4

Shier, H. (2001). Pathways to participation: Openings, opportunities and obligations. A new model for enhancing children's participation in decision-making, in line with article 12.1 of the United Nations Convention on the Rights of the Child. Children \& Society, 15, 107-117. doi: 10.1002/chi.617

Sinclair, R. (2004). Participation in practice: Making it meaningful, effective and sustainable. Children \& Society, 18, 106-118. doi: 10.1002/CHI.817

Soares, N. F. (2006). A investigação participativa no grupo social da infância. Currículo sem Fronteiras, 6(1), 25-40.

Stipek, D., \& Byler, P. (1997). Early childhood education teachers: Do they practice what they preach? Early Childhood Research Quarterly, 12, 305-325.doi:10.1016/ S0885-2006(97)90005-3

Tarman, B. (2012). Prospective teachers' beliefs and perceptions about teaching as a profession. Educational Sciences: Theory \& Practice, 12(3), 1964-1973.

Tobin, J. (2005). Quality in early childhood education: An anthropologist's perspective. Early Education \& Development, 16, 421-434.

Tomás, C. (2007). Há muitos mundos no mundo... Direitos das Crianças, cosmopolitismo infantil e movimentos sociais de crianças - Diálogos entre crianças de Portugal e Brasil (Tese de doutoramento). Instituto de Estudos da Criança/Universidade do Minho, Braga. 
UNICEF - Convenção Sobre os Direitos da Criança, 20 de novembro, 1989, Nações Unidas, Treaty Series, vol. 1577, p. 3. Disponível em: https:// www.unicef.pt/docs/pdf_publicacoes/convencao_direitos_crianca2004.pdf

Wen, X., Elicker, J. G., \& McMullen, M. B. (2011). Early childhood teachers' curriculum beliefs: Are they consistent with observed classroom practices? Early Education \& Development, 22(6), 945-969. doi:10.1080/10409289.2010.507495

\section{Legislação consultada}

Lei n. ${ }^{\circ}$ 4/97, de 10 de fevereiro. Diário da República n. ${ }^{\circ} 34$ - I Série - A. Retirado de: http://infancia.no.sapo.pt/docs/5-97.pdf 


\title{
IMPLEMENTATION OF TEACHERS' PARTICIPATION IN EARLY CHILDHOOD EDUCATION: TEACHER'S PERCEPTIONS
}

\begin{abstract}
The aim of this study was to analyse the relationship between observed quality in early childhood education and the promotion of participation, as well as to evaluate the psychometric properties of a measure designed to assess early childhood education teachers' perceptions about the degree of implementation of children's participation in early childhood education. 168 preschool teachers participated in this study, 40 of which were simultaneously observed, in their classrooms, with the Classroom Observation Scoring System - CLASS (Pianta, La Paro, \& Hamre, 2008). We identified two components of teachers' perceptions of children's participation: Children's Expression and Responsibility and Decision Making by the Adult. Decision Making by the Adult was negatively associated with the domains of classroom quality measured through CLASS and with teacher's educational level. Teachers from public centers scored higher in Children's Expression and Responsibility than teachers from private for-profit centers. Findings provide moderate evidence on the reliability and validity of the Assessment Questionnaire of Early Childhood Education Teachers' Perceptions about Children's Participation Right.
\end{abstract}

Keywords

Children's participation right; Questionnaire; Perceptions; Early childhood education

IMPLÉMENTATION DU DROIT DE PARTICIPATION DES ENFANTS EN CONTEXTE DE JARDIN D'ENFANCE: PERCEPTIONS DES ÉDUCATEURS

Résumé

Cette étude vise à étudier la relation entre la qualité observée en contexte de jardin d'enfance et la promotion de la participation, et aussi évaluer les 
caractéristiques psychométriques d'une mesure des perceptions des éducateurs concernant l'implémentation du droit de participation des enfants en ce contexte. Des 168 éducateurs participants, 40 ont été observés en contexte de salle à l'aide du Classroom Observation Scoring System (Pianta, La Paro, \& Hamre, 2008). Deux composantes sous-tendant les perceptions des éducateurs ont été identifiées: l'Expression et la Responsabilité des Enfants et la Prise de Décision par l'Adulte. La Prise de Décision par l'Adulte est négativement corrélée avec la qualité des salles et la formation scolaire des éducateurs. Les éducateurs du service public ont des scores plus élevés sur l'échelle d'Expression et de Responsabilité des Enfants que les éducateurs du service privé. Les résultats montrent que le Questionnaire d'Évaluation des Perceptions des Éducateurs du Droit de Participation des Enfants a des indices modérés de fiabilité et de validité.

Mots-clé

Droit de participation des enfants ; Questionnaire ; Perceptions ; Jardin d'enfance

Recebido em fevereiro/2015 Aceite para publicação em junho/2016

i Centro de Investigação e de Intervenção Social (CIS-UL), ISCTE - Instituto Universitário de Lisboa, Portugal.

ii Centro de Investigação e de Intervenção Social (CIS-UL), ISCTE - Instituto Universitário de Lisboa, Portugal.

iii Centro de Investigação e de Intervenção Social (CIS-UL), ISCTE - Instituto Universitário de Lisboa, Portugal.

Toda a correspondência relativa a este artigo deve ser enviada para: Cecília do Rosário da Mota Aguiar, ISCTE - Instituto Universitário de Lisboa, Escola de Ciências Sociais e Humanas, Departamento de Psicologia Social e das Organizações, Av. ${ }^{a}$ das Forças Armadas, 1649-026 Lisboa, Portugal. E-mail: cecilia.rosario.aguiar@iscte.pt 